Article

\title{
Phase Transitions in Equilibrium and Non-Equilibrium Models on Some Topologies
}

\author{
Francisco W. De Sousa Lima \\ Dietrich Stauffer Computational Physics Lab, Departamento de Física Universidade Federal do Piauí, \\ 64049-550 Teresina, PI, Brazil; fwslima@gmail.com; Tel.: +55-86-9414-4591 \\ Academic Editor: Giorgio Sonnino \\ Received: 23 October 2015; Accepted: 22 February 2016; Published: 3 March 2016
}

\begin{abstract}
On some regular and non-regular topologies, we studied the critical properties of models that present up-down symmetry, like the equilibrium Ising model and the nonequilibrium majority vote model. These are investigated on networks, like Apollonian (AN), Barabási-Albert (BA), small-worlds (SW), Voronoi-Delaunay (VD) and Erdös-Rényi (ER) random graphs. The review here is on phase transitions, critical points, exponents and universality classes that are compared to the results obtained for these models on regular square lattices (SL).
\end{abstract}

Keywords: nonequilibrium; phase transition; Monte Carlo simulations

\section{Introduction}

Some equilibrium and non-equilibrium models were studied on regularity and non-regularity with a scale-free (SF) and small-worlds (SW) networks [1-15] to understand the critical properties of these models on some networks.

According to the criterion of Grinstein et al. [16], non-equilibrium spin systems with two states $( \pm 1)$ on square lattices (SL) may present the same critical exponents of the Ising model (IM) on SL [3]. This criterion was confirmed in some non-equilibrium models [17-22] on regular lattices, such as the majority vote (MV) model with states $( \pm 1)$ [17]. This presents a continuous phase transition with critical exponents $\beta, \gamma, v$, similar to those of the IM [3] in agreement with the criterion of Grinstein et al. [16]. Lima et al. [6] have studied MV on Voronoi-Delaunay (VD) random lattices. There, the obtained exponents differ from those on SL, in disagreement with the criterion by Grinstein et al. [16].

For a decade, the IM has been investigated on undirected Apollonian networks (UAN) $[23,24]$ and directed Barabási-Albert networks (DBA) [14,15], and it has been shown that, on these networks, the IM does not display a phase transition.

In this review, we discuss the Ising and MV model on normal (UBA, USW, undirected Erdös-Rényi (UER) and UAN), DBA, directed small-worlds (DSW) [10,25] and directed Erdös-Rényi (DER) graphs [26,27], undirected and directed Voronoi-Delaunay (UVD and DVD) random lattices [28,29] and directed Apollonian networks (DAN) [30]. Through Monte Carlo (MC) simulations, it was found that the MV model on these networks presents a continuous phase transition showing that the MV and the IM belong to different universality class, therefore contradicting the Grinstein criterion [16]. Here, we study the models mentioned above only through MC simulations. However, important analytical results may be found in [31-34]. 


\section{Model}

The MV model dynamics' evolution is as follows. Initially, we have a spin variable $\sigma= \pm 1$ at each node or site of the network. At each MC time step, we try to spin flip a site. This is accepted with probability:

$$
w_{i}(\sigma)=\frac{1}{2}\left[1-(1-2 q) \sigma_{i} S\left(\sum_{\delta=1}^{k_{i}} \sigma_{i+\delta}\right)\right]
$$

$S(x)$ is a sign function with $S(x)= \pm 1$ of $x$ if $x \neq 0 ; S(x)=0$ if $x=0$. In the $w_{i}$ probability, the sum runs over the number $k_{i}$ of neighbors of the $i$-th spin. The control parameter $0 \leq q \leq 1$ plays a role of the "social temperature", similar to the temperature in IM; the smaller the $q$, the greater is the probability of parallel aligning with the local majority.

To study the properties critical for the MV model, we define the variable $m=\sum_{i=1}^{N} \sigma_{i} / N$. Here, we are interested in the magnetization $M$, susceptibility $\chi$ and the reduced fourth-order cumulant $U_{4}$ :

$$
\begin{gathered}
M=\langle|m|\rangle_{\mathrm{av}}, \\
\chi=N\left(\left\langle m^{2}\right\rangle_{\mathrm{av}}-\langle m\rangle_{\mathrm{av}}^{2}\right), \\
U_{4}=1-\left\langle m^{4}\right\rangle_{\mathrm{av}} / 3\left\langle m^{2}\right\rangle_{\mathrm{av}}^{2},
\end{gathered}
$$

where $\langle\cdots\rangle$ stands for a thermodynamics average. The results are averaged over the $R$ (av) networks' independent realizations. These physical quantities are functions of $q$ and obey the finite-size scaling relations (FSS):

$$
\begin{gathered}
M=N^{-\beta / v} f_{m}(t), \\
\chi=N^{\gamma / v} f_{\chi}(t), \\
\frac{d U}{d q}=N^{1 / v} f_{U}(t),
\end{gathered}
$$

where $1 / v, \beta / v$ and $\gamma / v$ are the critical exponents' ratios, and $f_{i}(t)$ are the FSS functions with:

$$
t=\left(q-q_{c}\right) N^{1 / v}
$$

being the scaling variable. From this scaling relation, we obtained the exponents $\beta / v$ and $\gamma / \nu$, respectively. Moreover, the value of $q^{*}$ for which $\chi$ has a maximum is expected to scale with the system size as:

$$
q^{*}=q_{c}+b N^{-1 / v},
$$

where $b=1$. The relation Equations (7) and (9) may be used to obtain the exponent $1 / v$. The MV model has also been studied in complex structures. Some of these structures will be described in the next section.

\section{Lattices, Graphs and Networks}

\section{- UAN and DAN}

The AN have $N=3+\left(3^{n}-1\right) / 2$ nodes $(N)$, and $n$ represents the generation number $[23,24]$. On these AN, we redirect a fraction $p$ of the links. This procedure results in a directed network, keeping the outgoing node of the redirected link, but changing the incoming node. If $p=0$, we have the standard AN, and for $p=1$, we have random networks [7]. However, there is the reciprocity of the redirected link in the undirected case, i.e., if Node $A$ selects Node $B$ as the incoming neighbor, then $A$ is also an incoming neighbor of $B$. 
- USW and DSW networks

The DSW networks in two dimensions, studied here, were generated from an SL [10] and the other irregular triangulation (Delaunay triangulation) [29]. The disorder introduced on these SW networks is the same used on AN networks.

- UBA and DBA networks

To generate the DBA networks [14], each new node added to the network selects, with connectivity $z$, already existing nodes as neighbors influencing it; the recently-added node does not influence these neighbors. In the case of the UBA networks [13], the recently-added node does influence these neighbors.

- UER and DER random graphs

The ER random graphs [7] are constructed by connecting pairs of randomly-selected nodes with a probability $p=2 k / N(N-1)$ with $N$ nodes and $k$ links (bonds). The connectivity of a node $k_{i}=\sum_{j} l_{i j}$, where $l_{i j}=1$ or 0 , is defined as the total number of links connected to it. These links can be undirected or directed, as well.

- UVD and DVD random lattices

The construction of the UVD random lattice [28] for a given set of points in the plane is given as follows. For each point, we first determine the polygonal cell consisting of the region of space nearer to that point than to any other point. Whenever two such cells share an edge, they are considered as neighbors. From the Voronoi diagram, we can obtain the dual lattice by the following procedure. When two cells are neighbors, one draws a link between the two points located in the center of each cell. From the links, one obtains the triangulation of space that is called the Delaunay triangulation. The Delaunay triangulation is dual to the Voronoi diagram, in the sense that points correspond to cells, links to edges and triangles to the vertices of the Voronoi tessellation. The DVD random lattices [29] are constructed in the same way as the DAN.

\section{Results and Discussion}

\subsection{Apollonian Networks}

- The IM

Andrade et al. [23,24] studied the IM on the UAN. They obtained the thermodynamic and magnetic properties, but they found no evidence of a phase transition on UAN for the IM.

- The MV model

The MV model was studied on triangular AN networks by Lima et al. [30]. We found a continuous phase transition. The effect of the reconnection of the links of the network with a probability $p$ were also studied. Through MC simulations, the exponents' ratios $\gamma / v, \beta / v$ and $1 / v$ were obtained for values of reconnection probability $p=0.0,0.10 .2,0.3,0.4,0.5,0.6,0.7,0.8$ and 0.9 . The critical noise $q_{c}$ and $U^{*}$ were also determinated. Here, the effective dimensionality of the system was observed to be independent of $p$, and its value $D_{\text {eff }}=2 \beta / v+\gamma / v \approx 1.0$ is observed for these networks.

Figure 1 displays the magnetization $M$, Binder's cumulant $U_{4}$ and susceptibility $v$ s. $q$, obtained from MC simulations on AN for $N=367,1096,3283,9844,29,527,88,576$ and 265,723 sites and for $g_{n}$ generation $(n=6,7,8,9,10,11$ and 12). The shape of the quantities' curves suggests the existence of the continuous phase transition in these networks. The values of $q_{c}$ is estimated as the point where the curves for different system sizes $N$ intercept each other [35]. In Table 1, we summarize the values of $q_{c}$ from $p=0.0$ to 0.9 . 


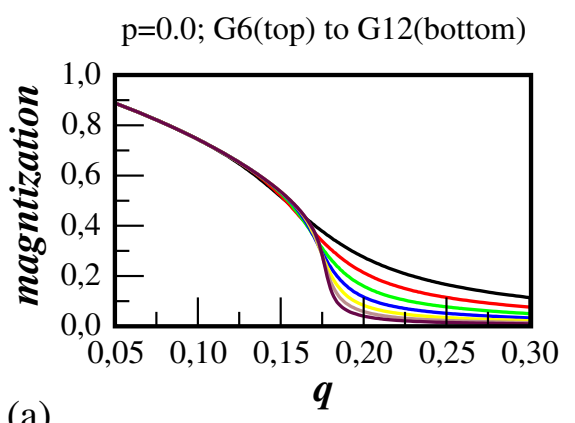

(a)

$\mathrm{p}=0.1$; G6(top) to G12(bottom)

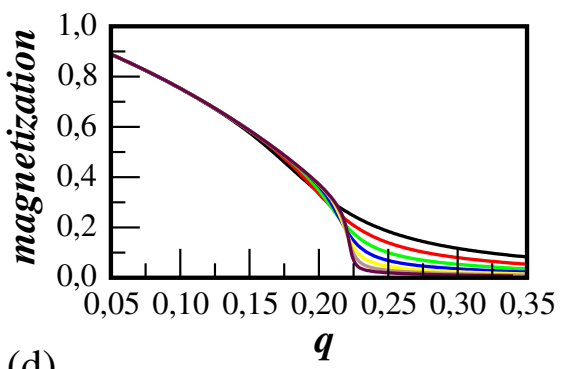

(d)

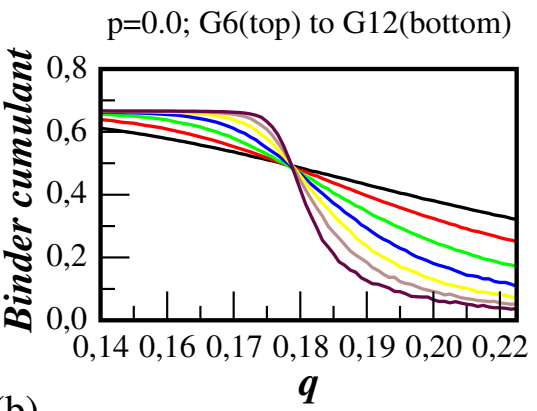

(b)

$\mathrm{p}=0.1$; G6(top) to G12(bottom)

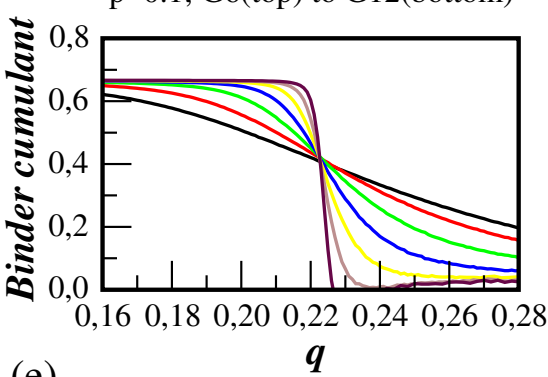

(e)

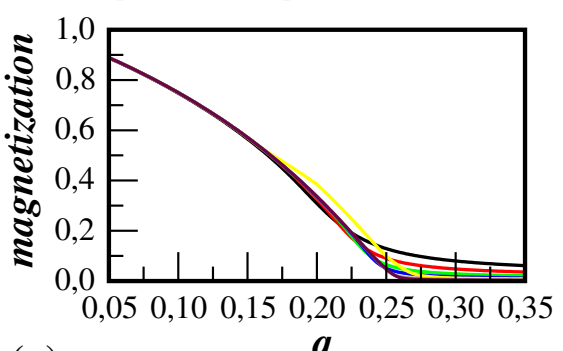

(g) $\mathrm{p}=0.9$; G6(top) to G12(bottom)

(h)

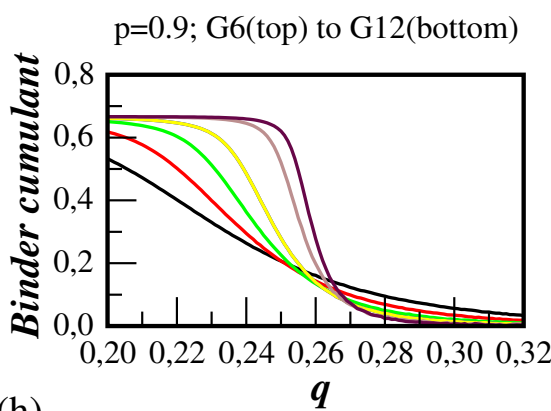

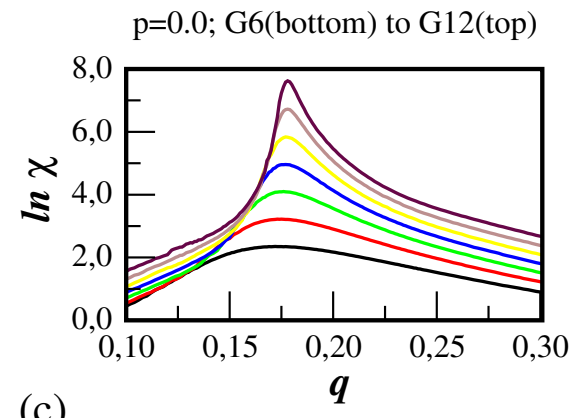

(c)

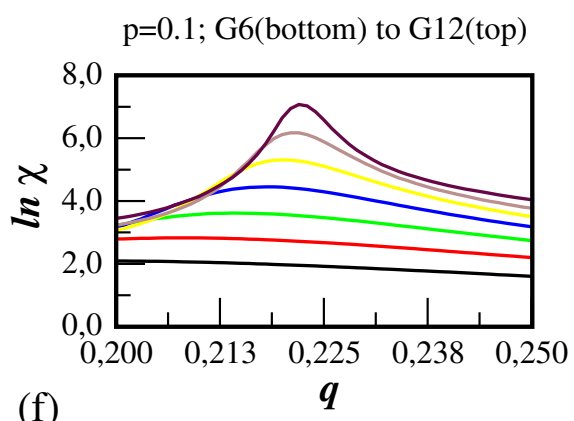

(f)

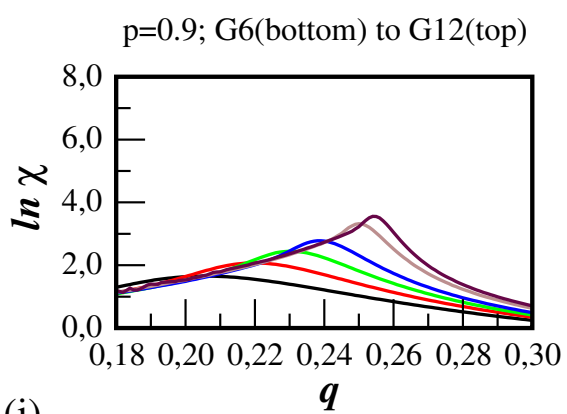

(i)

Figure 1. The magnetization $M(\mathbf{a}, \mathbf{d}, \mathbf{g})$, Binder's cumulant $U_{4}(\mathbf{b}, \mathbf{e}, \mathbf{h})$ and susceptibility $(\mathbf{c}, \mathbf{f}, \mathbf{i})$ vs. noise parameter $q$ for $N=367,1096,3283,9844,29,527,88,576$ and 265,723 sites and for $g_{n}$ generations $(n=6,7,8,9,10,11$ and 12) on an Apollonian (AN) network. We use reconnection probability from $p=0.0$ (undirected AN $(\mathrm{UAN}))(\mathbf{a}, \mathbf{b}, \mathbf{c}), p=0.1(\mathbf{d}, \mathbf{e}, \mathbf{f})$ and $p=0.9($ directed AN $(\mathrm{DAN}))(\mathbf{g}, \mathbf{h}, \mathbf{i})$ 
Table 1. The critical noise parameter $q_{c}$ and the critical exponents, for AN with reconnection probability $p$ [30]. Error bars are statistical only.

\begin{tabular}{cccccc}
\hline $\boldsymbol{p}$ & $\boldsymbol{q}_{\boldsymbol{c}}$ & $\mathbf{1} \boldsymbol{v}$ & $\boldsymbol{\beta} / \boldsymbol{v}$ & $\gamma / \boldsymbol{v}$ & $\boldsymbol{D}_{\text {eff }}$ \\
\hline 0.0 & $0.178(3)$ & $0.53(4)$ & $0.091(3)$ & $0.80(2)$ & $0.98(3)$ \\
0.1 & $0.223(5)$ & $0.48(2)$ & $0.097(5)$ & $0.79(3)$ & $0.98(3)$ \\
0.2 & $0.249(3)$ & $0.66(3)$ & $0.112(3)$ & $0.80(3)$ & $1.02(2)$ \\
0.3 & $0.271(5)$ & $0.61(8)$ & $0.148(5)$ & $0.72(5)$ & $1.02(3)$ \\
0.4 & $0.284(5)$ & $0.71(7)$ & $0.130(3)$ & $0.69(4)$ & $0.95(6)$ \\
0.5 & $0.296(4)$ & $0.44(5)$ & $0.220(8)$ & $0.55(3)$ & $0.99(5)$ \\
0.6 & $0.313(3)$ & $0.23(3)$ & $0.343(4)$ & $0.32(2)$ & $1.01(3)$ \\
0.7 & $0.311(5)$ & $0.21(5)$ & $0.374(5)$ & $0.25(3)$ & $1.01(4)$ \\
0.8 & $0.290(5)$ & $0.27(3)$ & $0.36(2)$ & $0.28(3)$ & $1.00(2)$ \\
0.9 & $0.2629(3)$ & $0.29(6)$ & $0.347(9)$ & $0.29(2)$ & $0.98(5)$ \\
\hline
\end{tabular}

In Figure 2, we plot the magnetization $M^{*}=M\left(q_{c}\right)$ vs. $N$. The fits of the curves correspond to the exponents' ratio $\beta / v$ according to relation Equation (5); see Table 1.

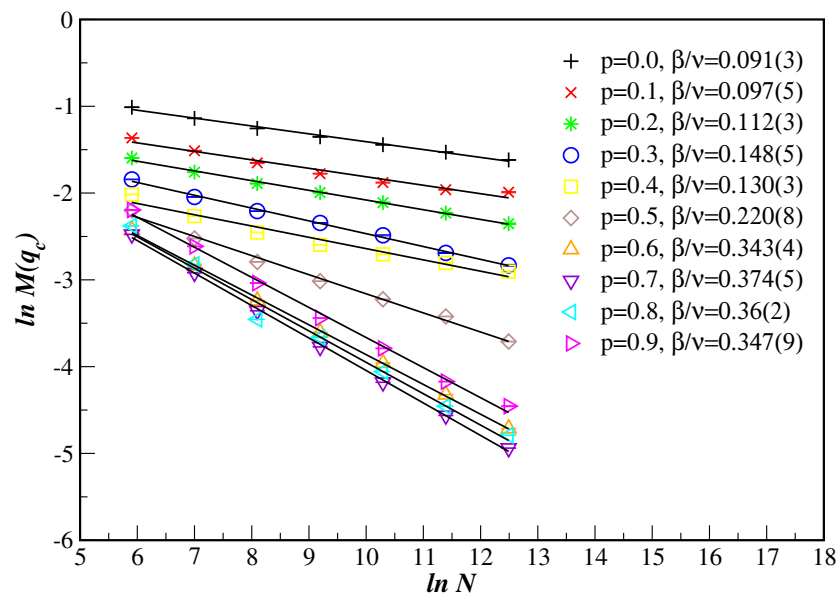

Figure 2. Plot of the $\ln M\left(q_{c}\right)$ vs. $\ln N$ for $p$-values from $p=0.0$ to 0.9 .

In Figure 3, we plot the susceptibility $\chi(N)$ at $q=q_{c}$ vs. $N$ for AN obtained from the relation Equation (6). The exponents' ratio $\gamma / v$ is obtained from the slopes of the straight lines for several values of the reconnection probability from $p=0.0$ to 0.9 .

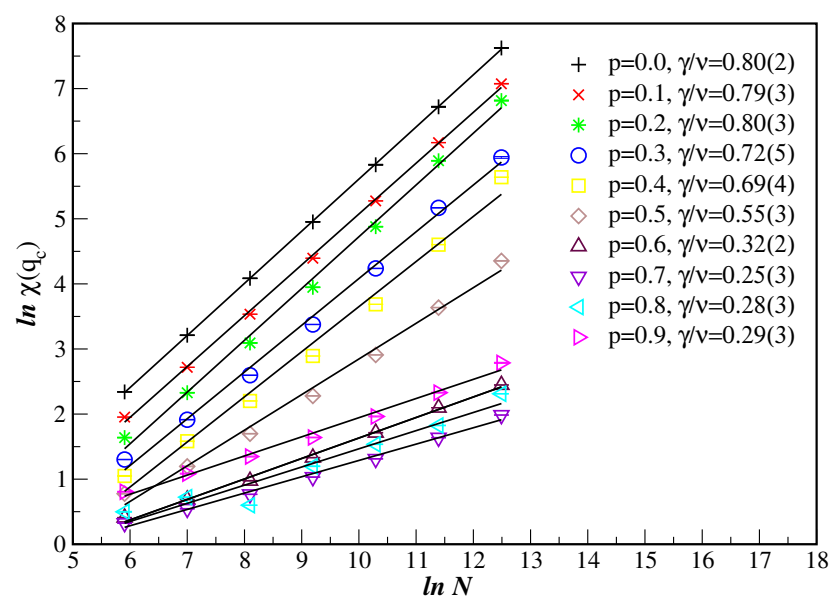

Figure 3. Plot of $\ln \chi(N)$ vs. $\ln N$ for some values of the reconnection probability from $p=0.0$ to 0.9 . 
In Figure 4, we used the scaling relation Equation (9) and obtain the exponents' ratio $1 / v$. The estimated values of the exponents $1 / v$ are in Table 1 . The results obtained by Andrade $e t$ al. $[23,24]$ on the IM in UAN have shown no phase transition existence. However, the results presented for the MV model on UAN demonstrate that this belongs to a different universality class than the IM on UAN; see Table 1.

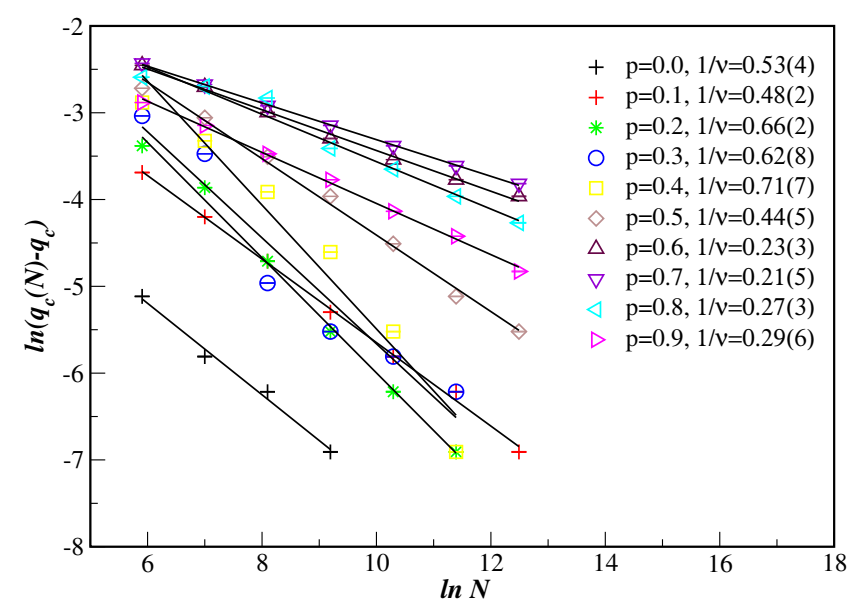

Figure 4. The exponents 1/v obtained from the relation Equation (9) for AN.

\subsection{ER Random Graphs}

- The IM

The IM was studied on independent ER graphs and different connectivities through MC simulations [26]. This model exhibits a phase transition-like mean-field, and the critical exponents on both DER and UER graphs are identical to the mean-field.

- $\quad$ The MV model

Through MC Simulation, the MV model was studied with noise on UER and DER random graphs $[27,36]$. Unlike IM, it presents a continuous phase transition on both DER and UER random graphs. The points $q_{c}$ and critical exponents' ratios $\beta / v, \gamma / v$ and $1 / v$ as a function of the connectivity $z$ of the random graphs have been obtained; see Table 2 .

Table 2. The noise $q_{c}$ and the critical exponents, for directed Erdös-Rényi (DER) random graphs with connectivity $z$ [27].

\begin{tabular}{ccccccc}
\hline $\boldsymbol{z}$ & $\boldsymbol{q}_{c}$ & $\beta / v$ & $\gamma / v^{\boldsymbol{q}_{c}}$ & $\gamma / v^{\boldsymbol{q}_{c}(N)}$ & $\mathbf{1} / \boldsymbol{v}$ & $\boldsymbol{D}_{\text {eff }}$ \\
\hline 4 & $0.175(4)$ & $0.230(5)$ & $0.530(6)$ & $0.516(2)$ & $0.545(26)$ & $0.990(7)$ \\
6 & $0.238(3)$ & $0.243(4)$ & $0.509(4)$ & $0.511(2)$ & $0.488(16)$ & $0.995(5)$ \\
8 & $0.274(3)$ & $0.238(4)$ & $0.512(4)$ & $0.504(2)$ & $0.548(14)$ & $0.988(5)$ \\
10 & $0.299(2)$ & $0.268(4)$ & $0.473(5)$ & $0.495(1)$ & $0.487(10)$ & $1.009(6)$ \\
20 & $0.359(2)$ & $0.280(4)$ & $0.451(4)$ & $0.485(2)$ & $0.510(10)$ & $1.011(5)$ \\
50 & $0.412(2)$ & $0.282(3)$ & $0.441(2)$ & $0.466(5)$ & $0.484(11)$ & $1.005(3)$ \\
100 & $0.438(2)$ & $0.286(2)$ & $0.428(3)$ & $0.440(8)$ & $0.520(19)$ & $1.000(3)$ \\
\hline
\end{tabular}

\subsection{BA Networks}

- The IM

The IM on UBA was first studied by Aleksiejuk et al. [13]. Through MC simulations [13], they showed that the critical temperature increases logarithmically with increasing system size $N$. Later, Sumour et al. [14,15] studied the IM on a DBA network. Unlike the results found by 
Aleksiejuk et al. [13], they showed that the IM on a DBA network does not present a phase transition.

- $\quad$ The MV model

The MV model was studied on DBA and UBA through MC simulations by Lima [21]. Their results obtained on DBA for the MV model show a clear transition continuous phase for values of $q_{c}$ dependent on $z$ neighbors; see Tables 3 and 4 .

Table 3. The connectivity $z$, critical noise parameter $q_{c}$, the critical exponents ratio, and the effective $D_{\text {eff }}$ for directed Barabási-Albert (DBA) networks [21].

\begin{tabular}{cccccc}
\hline $\boldsymbol{z}$ & $q_{c}$ & $\beta / v$ & $\gamma / v^{q_{c}}$ & $\gamma / v^{q_{c}(N)}$ & $D_{\text {eff }}$ \\
\hline 2 & $0.434(3)$ & $0.477(2)$ & $0.897(12)$ & $0.895(10)$ & $1.018(9)$ \\
3 & $0.431(4)$ & $0.444(1)$ & $0.905(15)$ & $0.904(12)$ & $0.999(2)$ \\
4 & $0.431(3)$ & $0.447(1)$ & $0.889(3)$ & $0.888(9)$ & $0.998(3)$ \\
6 & $0.438(2)$ & $0.435(2)$ & $0.863(5)$ & $0.861(3)$ & $1.008(6)$ \\
8 & $0.444(5)$ & $0.431(1)$ & $0.860(7)$ & $0.851(5)$ & $1.000(2)$ \\
10 & $0.446(3)$ & $0.421(2)$ & $0.831(5)$ & $0.834(7)$ & $1.000(5)$ \\
20 & $0.458(4)$ & $0.412(1)$ & $0.793(13)$ & $0.795(11)$ & $1.002(2)$ \\
50 & $0.467(2)$ & $0.375(4)$ & $0.715(11)$ & $0.735(17)$ & $0.999(11)$ \\
100 & $0.474(3)$ & $0.363(4)$ & $0.654(13)$ & $0.674(23)$ & $0.999(9)$ \\
\hline
\end{tabular}

Table 4. The connectivity $z$, critical noise parameter $q_{c}$, the critical exponents ratio and the effective $D_{\text {eff }}$ for UBA networks [37].

\begin{tabular}{ccccccc}
\hline $\boldsymbol{z}$ & $q_{c}$ & $\beta / v$ & $\gamma / v^{q_{c}}$ & $\gamma / v^{\boldsymbol{q}_{c}(N)}$ & $\mathbf{1} / \boldsymbol{v}$ & $\boldsymbol{D}_{\text {eff }}$ \\
\hline 2 & $0.167(3)$ & $0.036(8)$ & $0.828(6)$ & $0.805(11)$ & $0.76(3)$ & $0.90(1)$ \\
3 & $0.259(2)$ & $0.133(21)$ & $0.713(18)$ & $0.655(31)$ & $0.83(7)$ & $0.979(27)$ \\
4 & $0.306(3)$ & $0.231(22)$ & $0.537(8)$ & $0.519(17)$ & $0.43(2)$ & $0.999(23)$ \\
6 & $0.355(2)$ & $0.283(8)$ & $0.445(15)$ & $0.423(3)$ & $0.35(5)$ & $1.011(17)$ \\
8 & $0.380(6)$ & $0.323(2)$ & $0.358(7)$ & $0.405(6)$ & $0.39(5)$ & $1.004(7)$ \\
10 & $0.396(3)$ & $0.338(2)$ & $0.324(2)$ & $0.380(3)$ & $0.324(5)$ & $1.000(2)$ \\
20 & $0.428(2)$ & $0.334(2)$ & $0.305(2)$ & $0.350(2)$ & $0.307(5)$ & $0.993(2)$ \\
50 & $0.456(3)$ & $0.366(2)$ & $0.255(2)$ & $0.341(3)$ & $0.30(1)$ & $0.987(2)$ \\
100 & $0.471(3)$ & $0.373(2)$ & $0.218(5)$ & $0.330(3)$ & $0.308(3)$ & $0.964(5)$ \\
\hline
\end{tabular}

\subsection{SW Networks}

- $\quad$ The IM

The one-dimensional IM was studied, via MC simulations, on SW networks by Jeong et al. [38]. Their results are different from [39-44]. Their critical exponents are smaller than the exponents of the IM at two dimensions. However, for two- and three-dimensional models $[45,46]$ by MC simulations, it has been verified that the phase transition presents a mean-field behavior [47].

- The MV model

Through MC simulations, Luz and Lima [25] studied the MV model with noise $q$ on DSW networks, please see the Fortran program for the majority vote on small-world networks (2D) in Appendix. They calculated the critical noise parameter $q_{c}$ for reconnection probability $p=0.1$, $0.3,0.5,0.8$ and 1.0 of the DSW networks. Table 5 shows the reconnection probability, $q_{c}$, the exponents' ratio $\beta / \nu, \gamma / v$ and $1 / \nu$ for the DSW network. The results obtained show that the critical exponents of the MV model belong to different universality classes from Oliveira [17] on SL, of Pereira et al. [48] for UER random graphs, Lima [49] and Campos et al. [36] on USW networks. 
Table 5. The critical noise parameter $q_{c}$, the critical exponents ratio and probability $p$ for directed small-worlds (DSW) networks [25].

\begin{tabular}{cccccc}
\hline$p$ & $q_{c}$ & $\beta / v$ & $\gamma / v^{q_{c}}$ & $\gamma / v^{q_{c}(L)}$ & $\mathbf{1} / v$ \\
\hline 0.1 & $0.122(3)$ & $0.423(17)$ & $1.178(13)$ & $1.214(39)$ & $0.837(223)$ \\
0.3 & $0.149(3)$ & $0.419(21)$ & $1.148(5)$ & $1.152(28)$ & $1.059(208)$ \\
0.5 & $0.160(2)$ & $0.441(12)$ & $1.116(5)$ & $1.120(25)$ & $1.010(52)$ \\
0.8 & $0.164(2)$ & $0.436(9)$ & $1.149(5)$ & $1.117(23)$ & $1.248(158)$ \\
1.0 & $0.165(2)$ & $0.415(18)$ & $1.139(8)$ & $1.122(25)$ & $1.032(81)$ \\
\hline
\end{tabular}

\subsection{VD Random Lattices}

\section{- $\quad$ The IM}

The IM has been studied, via MC simulations, on UVD random lattices by Espriu et al. [50] using the local update algorithms, like Metropolis. Their results showed evidence that IM on UVD random lattices has the same critical behavior of the IM on SL. Posteriorly, Janke et al [28,51], using a global MC update algorithm [52], reweighting techniques [53] and finite size scaling analysis, studied the IM on UVD random lattices. Their results were similar to those found by Espriu et al. [50], showing that the IM on UVD random lattices belongs to the universality same class of the IM on SL. Thereafter, Lima et al. [54] have also studied this model with an exchange coupling $J(r)=J_{0} e^{-\alpha r}$ that varies with the distance $r$ between the first neighbors for $\alpha \geq 0$ and $J_{0}=1$. Their results showed that this random system also falls in the same universality class as the IM on SL.

The IM on a directed small-world Voronoi-Delaunay (DSWVD) network was also studied by Sousa and Lima [29]. These results show a strong indication that the IM on DSWVD random lattices is in a different universality class than the model on an SL. The exponents obtained are independent of $p(0<p<1)$ and different from the IM on SL; see Table 6.

Table 6. The critical exponents, for spin $1 / 2$ on a small-world Voronoi-Delaunay (DSWVD) random lattice with probability $p$ [29]. The $\gamma / \nu^{\max }$ are the results from the maximum of the magnetic susceptibility.

\begin{tabular}{ccccc}
\hline$p$ & $\beta / v$ & $\gamma / v$ & $\gamma / v^{\max }$ & $\mathbf{1} / v$ \\
\hline 0.1 & $0.489(8)$ & $1.003(11)$ & $1.001(13)$ & $1.036(49)$ \\
0.2 & $0.538(68)$ & $1.016(11)$ & $1.016(5)$ & $1.098(82)$ \\
0.3 & $0.463(4)$ & $0.924(98)$ & $1.012(3)$ & $1.009(49)$ \\
0.4 & $0.491(9)$ & $1.017(14)$ & $1.012(8)$ & $0.886(8)$ \\
0.5 & $0.494(10)$ & $0.998(18)$ & $1.005(66)$ & $0.987(64)$ \\
0.6 & $0.486(10)$ & $1.042(13)$ & $1.004(7)$ & $0.927(92)$ \\
0.7 & $0.486(10)$ & $1.016(13)$ & $1.003(10)$ & $1.107(60)$ \\
0.8 & $0.493(16)$ & $1.018(23)$ & $1.021(7)$ & $0.972(57)$ \\
0.9 & $0.471(12)$ & $1.038(16)$ & $0.991(69)$ & $1.032(66)$ \\
\hline
\end{tabular}

- The MV model

Lima et al. [6] studied the MV model on VD random lattices. These present a quenched disorder in their links. They investigated whether only this type of disorder is relevant to obtain critical exponents different from those found for the MV model on SL that have the same exponents of the IM on SL. They found the critical exponents' ratios $1 / v=0.99(8), \beta / \nu=0.112(4)$ and $\gamma / \nu=1.51(04)$. Therefore, they showed that critical exponents' ratios $\beta / \nu$ and $\gamma / \nu$ are different from the exact values of the IM and MV model on SL. 


\section{Conclusions}

We presented results for the equilibrium Ising and non-equilibrium MV models on AN, BA and SW networks, ER random graphs and the VD random lattice. On these networks, the non-equilibrium MV model shows a continuous phase transition. On the other hand, the IM does not have a phase transition on UAN and DBA networks $[14,15,23,24]$. Therefore, these results demonstrate that the MV model on UBA, DBA, UAN, DAN, DSW, UER, DER and DSWVD networks belongs to different universality classes, in disagreement with the criterion of Grinstein et al. [16]. A possible explanation for this different behavior may be attributed to the behavior of the critical points of these models, $q$ and $T$. In the IM, the flip probability of a spin (highly connected) against your neighborhood is smaller than for a less connected spin. Therefore, in the IM, the variation of energy is higher for a more connected spin. However, in the MV model, the flip probability of a spin against your neighborhood is always given by $q$, and it does not depend on the neighborhood of this spin. Interestingly, the effective dimensionality of the MV on DAN, UAN, UBA, DBA, UER and DER networks, defined as $D_{\text {eff }}=2 \beta / v+\gamma / v$, is always a value close to 1.0 , independent of the reconnection probability $p$, as seen in Tables $1-4$.

Acknowledgments: Francisco W. De Sousa Lima thanks Dietrich Stauffer for many suggestions and fruitful discussions during the development of this work. We thank Brazilian agency (CNPq) for financial support. This work also was supported by system Silicon Graphics Internacional (SGI) Altix 1350 (CENAPAD.UNICAMP-USP, SP-BRAZIL).

Conflicts of Interest: The authors declare no conflict of interest.

\section{Appendix: MV Fortran Program for DSW Starting from a Square Lattice}

This is the Fortran program for the majority vote on small-world networks (2D) (11/02/2011).

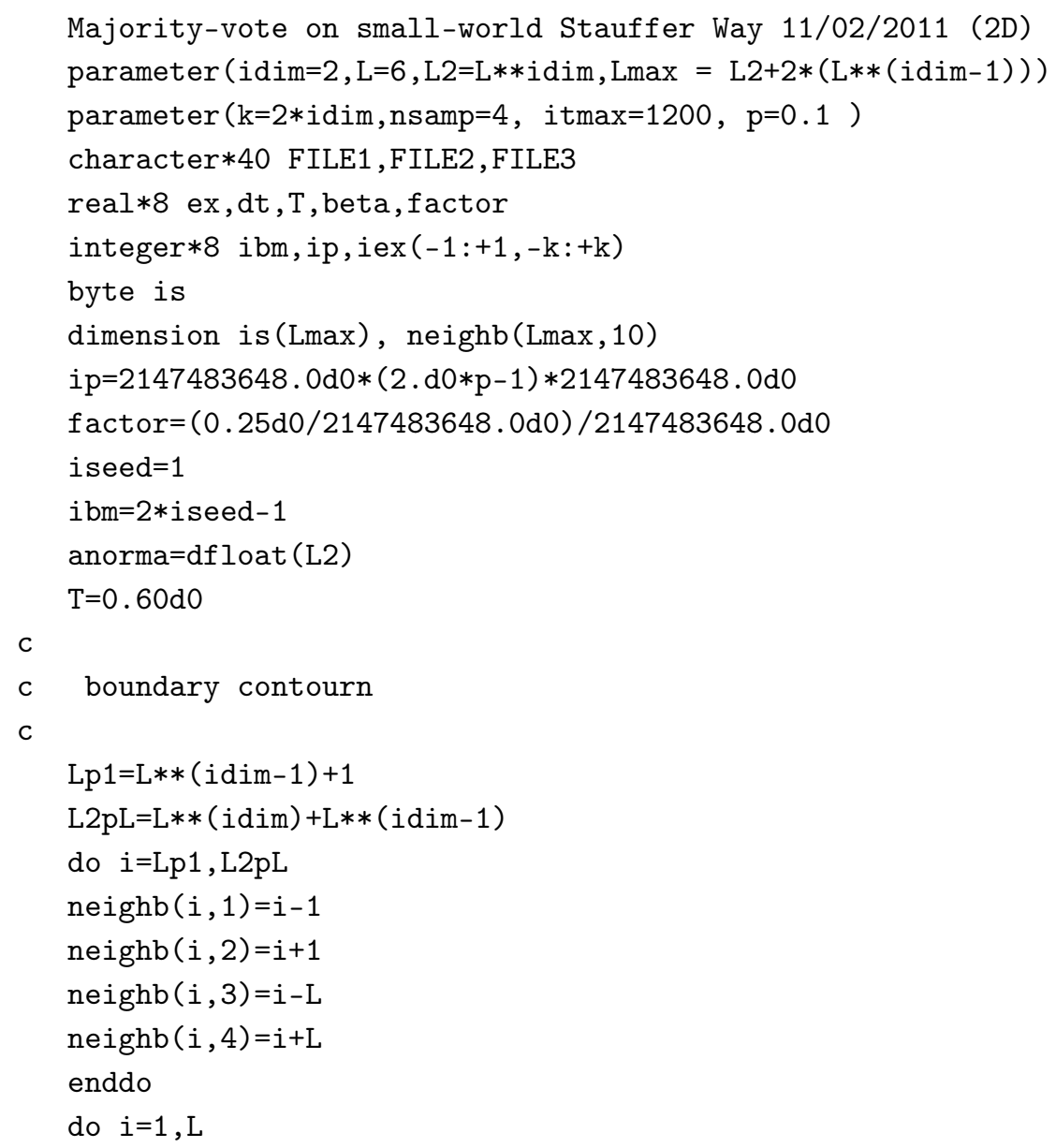




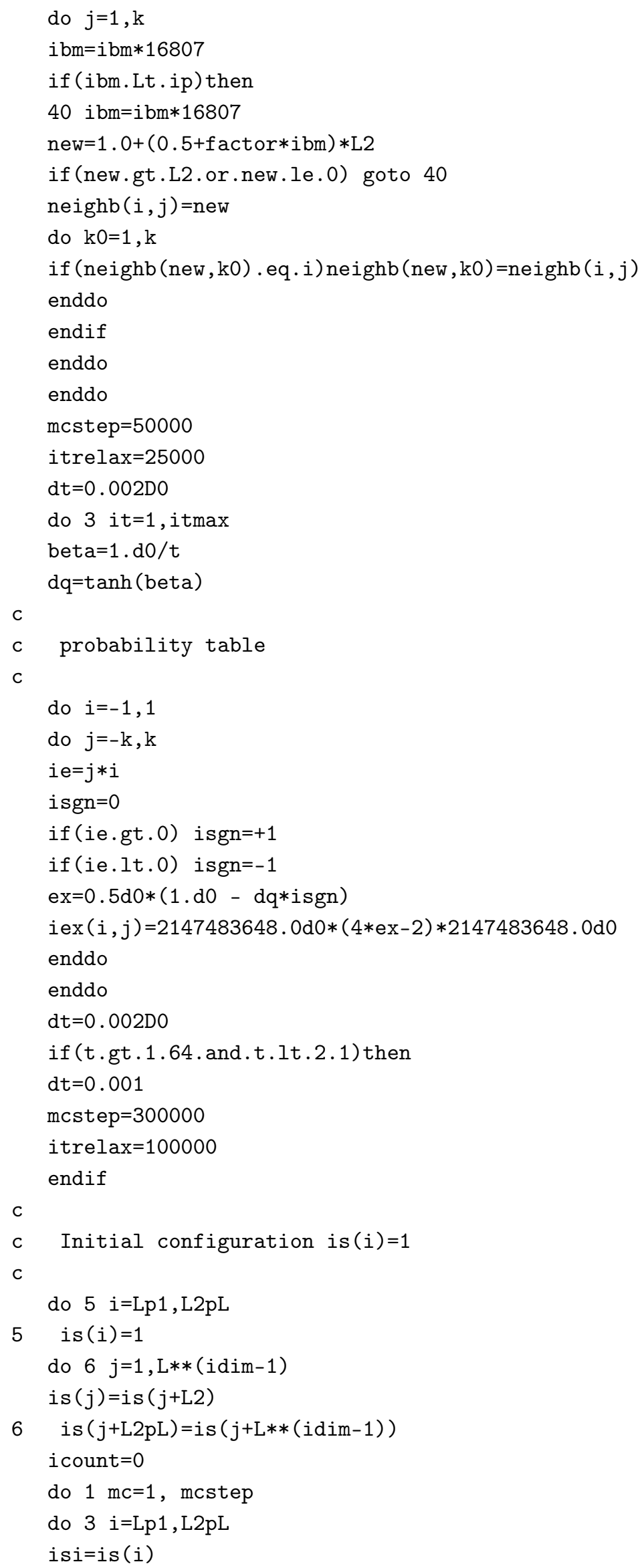




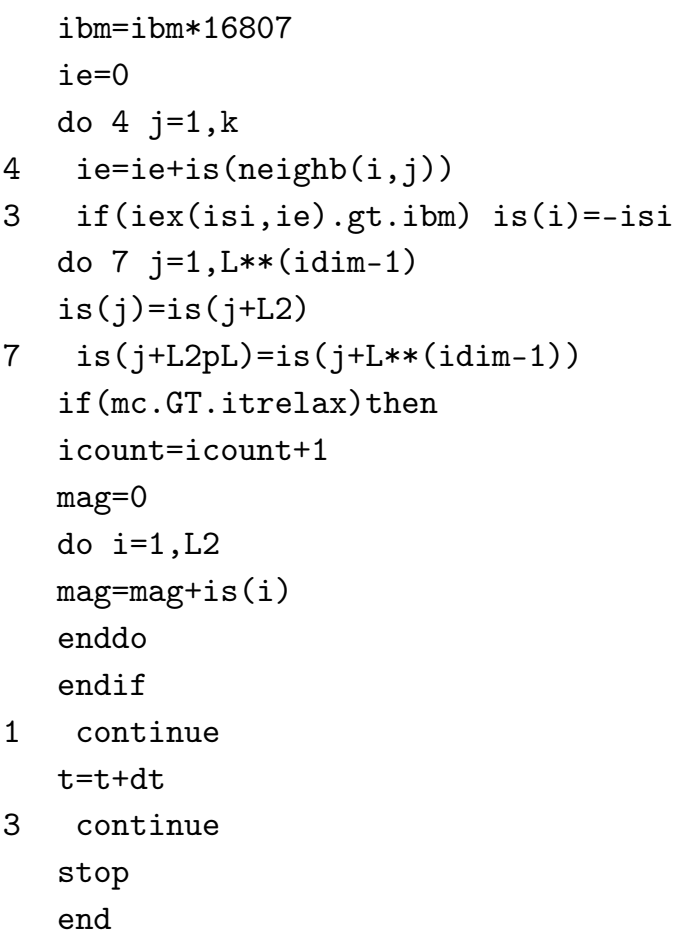

\section{References}

1. Lenz, W. Beiträge zum Verständnis der magnetischen Eigenschaften in festen Körpern. Physikalische Zeitschrift 1921, 21, 613-615.

2. Ising, E. Beitrag zur Theorie des Ferromagnetizmus. Zeitschrift für Physik 1925, 31, 253-258.

3. Baxter, R.J. Exactly Solved Models in Statistical Mechanics; Academic Press: London, UK, 1982.

4. Metropolis, N.; Rosenbluth, A.W.; Rosenbluth, M.N.; Teller, A.H.; Teller, E. Equation of State Calculations by Fast Computing Machines. J. Chem. Phys. 1953, 21, 1087-1092.

5. Lima, F.W.S.; Stauffer, D. Ising model simulation in directed lattices and networks. Physica A 2006, 359, 423-429.

6. Lima, F.W.S.; Fulco, U.L.; Costa Filho, R.N. Majority-vote model on a random lattice. Phys. Rev. E 2005, 71, 036105.

7. Erdös, P.; Rényi, A. On random graphs I. Publ. Math. Debrecen 1959, 6, 290-297.

8. Barrat, A.; Weigt, M. On the properties of small-world network models. Eur. Phys. J. B 2000, 13, 547-560.

9. Newman, M.E.J.; Strogatz, S.H.; Watts, D.J. Random graphs with arbitrary degree distributions and their applications. Phys. Rev. E 2001, 64, 026118.

10. Sanchez, A.D.; Lopez, J.M.; Rodriguez, M.A. Nonequilibrium phase transitions in directed small-world networks. Phys. Rev. Lett. 2002, 88, 048701.

11. Barabási, A.-L.; Albert, R. Emergence of Scaling in Random Networks. Science 1999, 286, 509-512.

12. Albert, R.; Jeong, H.; Barabási, A.-L. Internet: Diameter of the world-wide web. Nature 1999, 401, 130-131.

13. Aleksiejuk, A.; Hołyst, J.A.; Stauffer, D. Ferromagnetic phase transition in Barabasi-Albert networks. Physica A 2002, 310, 260-266.

14. Sumour, M.A.; Shabat, M.M. Monte Carlo simulation of Ising model on directed Barabasi-Albert Network. Int. J. Mod. Phys. C 2005, 16, 585-589.

15. Sumour, M.A.; Shabat, M.M.; Stauffer, D. Absence of Ferromagnetism in Ising model on directed Barabasi-Albert network. Islam. Univ. J. 2006, 14, 209-212.

16. Grinstein, G.; Jayaprakash, C.; He, Y. Statistical Mechanics of Probabilistic Cellular Automata. Phys. Rev. Lett. 1985, 55, 2527, doi:10.1103/PhysRevLett.55.2527.

17. De Oliveira, M.J. Isotropic majority-vote model on a square lattice. J. Stat. Phys. 1992, 66, $273-281$.

18. Bennett, C.H.; Grinstein, G. Role of irreversibility in stabilizing complex and nonergodic behavior in locally interacting discrete systems. Phys. Rev. Lett. 1985, 55, 657, doi:10.1103/PhysRevLett.55.657. 
19. Wang, J.S.; Lebowitz, J.L. Phase transitions and universality in nonequilibrium steady states of stochastic Ising models. J. Stat. Phys. 1988, 51, 893-906.

20. Marques, M.C. Nonequilibrium Ising model with competing dynamics: A MFRG approach. Phys. Lett. A 1990, 145, 379-382.

21. Lima, F.W.S. Majority-vote on directed Barabasi-Albert networks. Int. J. Mod. Phys. C 2006, 17, 1257-1265.

22. Santos, J.C.; Lima, F.W.S.; Malarz, K. Majority-vote model on triangular, honeycomb and Kagomé lattices. Physica A 2011, 390, 359-364.

23. Andrade, R.S.F.; Herrmann, H.J. Magnetic models on Apollonian networks. Phys. Rev. E 2005, 71, 056131.

24. Andrade, R.S.F.; Andrade, J.S., Jr.; Herrmann, H.J. Ising model on the Apollonian network with node-dependent interactions. Phys. Rev. E 2009, 79, 036105.

25. Luz, E.M.S.; Lima, F.W.S. Majority-vote on directed small-world networks. Int. J. Mod. Phys. C 2007, 18, 1251-1261.

26. Lima, F.W.S.; Sumour, M.A. Ising model with spins $\mathrm{S}=1 / 2$ and 1 on directed and undirected Erdös-Rènyi random graphs. Physica. A 2012, 391, 948-953.

27. Lima, F.W.S.; Sousa, A.O.; Sumour, M.A. Majority-vote model on directed Erdös-Rènyi random graphs. Physica A 2008, 387, 3503-3510.

28. Janke, W.; Katoot, M.; Villanova, R. Ising model universality for two-dimensional lattices. Phys. Lett. B 1993, 315, 412-416.

29. Sousa, E.M.; Lima, F.W.S. Ising model on directed small-world Voronoi Delaunay random lattices. Eur. Phys. J. Plus 2013, 128, 150, doi:10.1140/epjp/i2013-13150-9.

30. Lima, F.W.S.; Moreira, A.A.; Araújo, A.D. Nonequilibrium model on Apollonian networks. Phys. Rev. E 2012, 86, 056109.

31. Sukhorukov, A.A.; Akhmediev, N.N. Intensity limits for stationary and interacting multi-soliton complexes. Phys. Lett. A 2002, 305, 160-166.

32. Dorogovtsev, S.N.; Goltsev, A.V. Critical phenomena in complex networks. Rev. Mod. Phys. 2008, 80, 1275, doi:10.1103/RevModPhys.80.1275.

33. Gleeson, J.P. Binary-State Dynamics on Complex Networks: Pair Approximation and Beyond. Phys. Rev. X 2013, 3, 021004.

34. Chen, H.; Shen, C.; He, G.; Zhang, H.; Hou, Z. Critical noise of majority-vote model on complex networks. Phys. Rev. E 2015, 91, 022816.

35. Binder, K.; Heermann, D. Monte Carlo Simulation in Statistical Phyics; Springer: Berlin/Heidelberg, Germany, 1988.

36. Campos, P.R.; de Oliveira, V.M.; Moreira, F.G.B. Small-world effects in the majority-vote model. Phys. Rev. E 2003, 67, 026104.

37. Lima, F.W.S. Majority-vote on Undirected Barabasi-Albert networks. Commun. Comput. Phys. 2007, 2, 358-366.

38. Jeong, D.; Hong, H.; Kim, B.J.; Choi, M.Y. Phase transition in the Ising model on a small-world network with distance-dependent interactions. Phys. Rev. E 2003, 68, 027101.

39. Gitterman, M. Small-world phenomena in physics: The Ising model. J. Phys. A 2000, 33, 8373, doi:10.1088/ 0305-4470/33/47/304.

40. Barrat, A.; Weight, M. On the properties of small-world network models. Eur. Phys. J. B 2000, 13, 547-560.

41. Pȩkalski, A. Ising model on a small world network. Phys. Rev. E 2001, 64, 057104.

42. Hong, H.; Kim, B.J.; Choi, MY. Comment on "Ising model on a small world network". Phys. Rev. E 2002, 66, 018101 .

43. Kim, B.J.; Hong, H.; Holme, P.; Jeon, G.S.; Minnhagen, P.; Choi, M.Y. XY model in small-world networks. Phys. Rev. E 2001, 64, 056135.

44. Novotny, M.A.; Wheeler, S.M. On the possibility of quasi small-world nanomaterials. Braz. J. Phys. 2004, 34, 395-400.

45. Zhang, Z. Critical Properties of Small World Ising models. Ph.D. Thesis, Mississippi State University, Starkville, MS, USA, 2005.

46. Richards, H.L.; Sides, S.W.; Novotny, M.A.; Rikvold, P.A. Kinetic Ising systems as models of magnetization switching in submicron ferromagnets. J. Appl. Phys. 1996, 79, 5749-5751.

47. Herrero, C.P. Ising model in small-world networks. Phys. Rev. E 2002, 65, 066110. 
48. Pereira, L.F.C.; Moreira, F.G.B. Majority-Vote model on random graphs. Phys. Rev. E 2005, 71, 016123.

49. Lima, F.W.S. Majority-vote on directed Barabasi-Albert networks. Int. J. Mod. Phys. C 2006, 7, 1257-1265.

50. Espriu, D; Gross, M.; Rakow, P.E.L.; Wheater, J.F. Random Lattices versus Regular Lattices. Prog. Theor. Phys. Suppl. 1986, 86, 304-321.

51. Janke, W.; Katoot, M.; Villanova, R. Single-cluster Monte Carlo study of the Ising model on two-dimensional random lattices. Phys. Rev. B 1994, 49, 9644, doi:10.1103/PhysRevB.49.9644.

52. Wolff, U. Collective Monte Carlo updating for spin systems. Phys. Rev. Lett. 1989, 62, 361, doi:10.1103/ PhysRevLett.62.361.

53. Ferrenberg, A.M.; Swendsen, R.H. New Monte Carlo technique for studying phase transitions. Phys. Rev. Lett. 1988, 61, 2635, doi:10.1103/PhysRevLett.61.2635.

54. Lima, F.W.S.; Moreira, J.E.; Andrade, J.S., Jr; Costa, U.M.S. The ferromagnetic Ising model on a Voronoi-Delaunay lattice. Physica A 2000, 283, 100-106.

(C) 2016 by the author; licensee MDPI, Basel, Switzerland. This article is an open access article distributed under the terms and conditions of the Creative Commons by Attribution (CC-BY) license (http:/ / creativecommons.org/licenses/by/4.0/). 